RESEARCH ARTICLE

\title{
Bioregulatory Role of Wickerhamomyces anomalus Killer Toxin against Pathogenic Members of the Genus Candida
}

\author{
Deepa Radhamany ${ }^{1}$, Sunil Rao $\mathrm{P}^{2}$, Subbannayya Kotigadde ${ }^{3}$ \\ ${ }^{1}$ Department of Microbiology, SR Medical College and Research Center, Akathumuri, Vettoor-cherunniyoor, Kerala, \\ India \\ ${ }^{2}$ Department of Microbiology, Mount Zion Medical College, Adoor, Kerala, India \\ ${ }^{3}$ K. V.G. Medical College \& Hospital, Sullia, Karnataka, India
}

\begin{abstract}
Objectives: Candida species are endogenous fungus causing both mucosal and invasive infections. With the increasing rate of non-albicans Candida infections with varying susceptibilities, early and accurate species identification would help the clinician in befitting therapeutic management. So the interest in natural products secreted by a fungus Wickerhamomyces anomalus itself as an antimicrobial agent against pathogenic Candida species has evaluated in the present study.
\end{abstract}

Methods: A total of 336 Candida species isolated from various clinical samples were included in the study. Candida identification, antifungal susceptibility was done by standard procedures. The environmental yeast killer toxin Wickerhamomyces anomalus was purified and the killer toxin assay against Candida species was done by standard procedures.

Results: The predominant Candida species were Candida albicans (31\%), but the emergence of non-albicans Candida also noted with 69\%. Total antifungal resistance was 117 (35\%) with fluconazole resistance in Candida albicans $13(13 \%)$ and in non-albicans Candida were $50(21 \%)$. The characterization of W. anomalus toxin has revealed that it is a glycoprotein of having $42.6 \mathrm{kDa}$ active at $\mathrm{pH} 5.5$ and temperatures $250 \mathrm{C}$. Candida isolates showed 292 (87\%) sensitivity to the killer toxin.

Conclusions: The study emphasizes the need for rapid and precise speciation and antifungal susceptibility of Candida isolates for effective treatment and management strategies.Our study proved the antifungal ability of $W$. anomalus and this resource can be used for alternative treatment of candidiasis with further assessments. J Microbiol Infect Dis 2019; 9(3):155-160.

Keywords: Candida albicans, Non-albicans Candida, Yeast killer toxin, Wickerhamomyces anomalus

\section{INTRODUCTION}

Candidiasis is an important opportunistic fungal infection of immunocompromised hosts with changing epidemiological trends [1]. The increased rate of immunosuppressive conditions during the last few decades, as a result of chemotherapy led to a parallel increase in the incidence of Candida albicans species and nonalbicans Candida species causing mucosal and systemic infections [2]. The emergence of antifungal resistant non-albicans Candida and the irrational use of antibiotics are also complicated the antifungal therapy of candidiasis. So the change in epidemiology and antifungal resistance has created the need of its speciation and susceptibility testing in selecting the antifungal agent for the treatment of Candida infections $[3,4]$.

Natural antifungal proteins are produced by a diverse group of organisms including bacteria, fungi, insects, vertebrates, invertebrates as well as plants. Yeast killer toxins are considered in this group and have been shown to have a broad spectrum of killing activity against various plant and human pathogens [5]. It has been reported that killer phenomenon is widely distributed and almost 100 yeast species belonging to more than 20 genera are killer toxin producers [6]. Among the killer yeasts $W$. anomalus has been found to have a potential

Correspondence: Dr. Deepa Radhamany, Department of Microbiology, SR Medical College and Research Center, Akathumuri, Vettoor-cherunniyoor, Kerala, India Email: deepababin@gmail.com

Received: 09 January 2019 Accepted: 10 November 2019

Copyright (C) JMID / Journal of Microbiology and Infectious Diseases 2019, All rights reserved 
killing effect on pathogenic and non-pathogenic fungus. The strong therapeutic activity exerted against different experimental mucosal and systemic mycoses by monoclonal and recombinant microbicidal killer toxin antibodies as well as by a synthetic killer peptide suggested new potential strategies for therapy $[7,8]$.So the need of a novel antifungal agent with less toxicity and wide spectrum of action from a natural source also pointing towards the use of killer toxins from yeasts. Indian studies on yeast killer toxin activity against pathogenic Candida species are scanty. Hence the present study was chosen to evaluate the anticandidial effect of yeast killer toxin produced by $W$. anomalus from environmental isolates.

\section{METHODS}

A total of $(n=336)$ Candida strains were isolated from patients attending both outpatient and inpatient departments of Travancore Medical College, Kollam. It was conducted over a period starting from August 2011 to August 2016. The study protocol was approved by the Institutional Ethical Committee of the Travancore Medical College Hospital, Kerala registered IEC. No:$001 / 11$.

Various clinical specimens like high vaginal swabs, blood, urine, aspirates, catheter devices, cerebrospinal fluid, oral scrapings, sputum and nail clippings received at the Microbiology laboratory for culture were included in the study. Samples from drainage tubes or catheter bags and Candida colony counts below $10^{2} \mathrm{CFU} / \mathrm{mL}$ of urine and sputum specimens were excluded.

\section{Phenotypic Identification of Candida isolates}

The Standard protocol for yeast identification included the following tests - $\mathrm{KOH}$ mount, Gram's stain, culture on Sabouraud's dextrose agar with chloramphenicol, germ tube test, urea hydrolysis, cornmeal agar morphology, sugar fermentation test, sugar assimilation test (Auxanographic Plate Method),CHROM agar morphology, and VITEK.

\section{Antifungal Susceptibility Testing}

Antifungal susceptibility testing of the Candida isolates was performed by micro broth dilution method in accordance with Clinical and Laboratory Standard institute (CLSI) guidelines M27-A3 [9].

\section{Antifungal agents}

Antifungal agents were used in powder forms amphotericin B (AMB- Sigma) fluconazole (FCZPfizer NY), 5-flucytosine (Sigma), voriconazole (VCZ- Pfizer NY), caspofungin (Merck \& Co., Inc) E strips were used only for blood isolates.

\section{Wickerhamomyces anomalus killer toxin assay}

$W$. anomalus was the environmental yeast killer toxin strain originally isolated from cashew leaves was used in the study. The strains were maintained on YEPD agar slants at $20{ }^{\circ} \mathrm{C}$. For the killer toxin production and purification $W$. anomalus strains were cultured in YEPD medium. YEPD broth medium supplemented $5 \%$ glycerol buffered with citrate phosphate to $\mathrm{pH}$ 4.5 was used for killer toxin production $[10,11]$.

\section{Purification of killer toxin}

The crude toxin of $W$. anomalus was subjected to ammonium sulphate precipitation (30-100\%), and each of the precipitated fraction was dissolved in citrate phosphate buffer $(50 \mathrm{mM}, \mathrm{pH}$ 5 ), and examined for killer activity and protein content by Lowry's method [12]. The eluted fraction after ammonium precipitation which showed killer activity were pooled, dialyzed, and subjected to gel chromatography using sephadex gel filtration G-10 $(5.5 \mathrm{~cm} \times 100 \mathrm{~cm})$ (Sigma Aldrich) [13].

\section{SDS-PAGE}

The active fraction obtained from HPLC was subjected to $5 \%-20 \%$ linear gradient SDSPAGE gel in a discontinuous buffer system under denaturing conditions. Sodium dodecyl sulphate (SDS)-PAGE analysis of purified killer toxin protein was done for determining its molecular weight [14].

\section{Agar Well Diffusion Assay}

$W$. anomalus killer yeast isolate was grown in yeast extract peptone dextrose medium (YEPD) for 72 hours [15].Crude toxin was prepared and poured into wells of diameter $7 \mathrm{~mm}$ (at $50 \mu \mathrm{l}$ per well) cut on yeast extract peptone dextrose medium with methylene blue. The plates prespread with Candida species. Plates were incubated at $30{ }^{\circ} \mathrm{C}$ for 24 hours, and zone of inhibition was measured. The killing activity of 
each sample was measured and defined as the mean of inhibition of three replicate wells.

\section{Optimum temperature and $\mathrm{pH}$ for Wickerhamomyces anomalus killer toxin}

The purified toxin was poured in the wells of YEPD plates pre-spread with sensitive Candida species and incubated at different temperatures $\left(20,25,30\right.$ and $\left.37^{\circ} \mathrm{C}\right)$, for $24-72$ hours and at different $\mathrm{pH}$ (3-7). The activity was determined by measuring the inhibition zone size [15].

\section{Statistical analysis}

Descriptive statistics were used for analysis. Chi-square test used for analysis of virulence factors, antifungal susceptibility and killer toxin assay ( $p$ value $<0.05$ was considered to be statistically significant).Statistical software SPSS (ver. 16, IL, Chicago) was used for data analysis. Measure of agreement between the virulence tests and killer toxin assay tests was estimated using cross tabulations. The significance level was set at a $p$ value $<0.05$.

\section{RESULTS}

Species distribution of Candida species from clinical specimens

A total of 336 Candida isolates were obtained from various clinical specimens during the period of study from 2011 to 2015 . A higher rate of $C$. albicans was found in the high vaginal swabs 41 (31\%) collected from women of reproductive age, urine 25 (24\%) and blood 11 (11\%) out of total 103 isolates. The predominant non-albicans Candida were C. tropicalis 87 (26 $\%)$ followed by C. krusei 71 (21\%), C. glabrata $(12 \%)$. A total of $40(12 \%)$ isolates were from blood, in which C. albicans (28\%) were the major isolate followed by C. tropicalis (23\%). Invasive device related Candida infections were $26(8 \%)$, which is associated with urinary catheters and central lines. The major isolate was C. tropicalis 7 (27\%).

\section{Antifungal Susceptibility by Micro-dilution Method}

Total antifungal resistance strains were 117 $(35 \%)$ out of 336 Candida isolates. Fluconazole resistance in C. albicans were $13(13 \%)$ and 50 $(21 \%)$ in non-albicans Candida isolates, except C. krusei because it is not tested for fluconazole as it is inherently resistant. Amphotericin-B and flucytosine resistance in C. albicans were 5 (5\%) and 7 (7\%). C. tropicalis got 27 (31\%) fluconazole resistances, $5(6 \%)$ resistance to flucytosine, $4(5 \%)$ resistance to voriconazole, and $3(3 \%)$ resistance to amphotericin B.

\section{Purification of killer toxin}

\section{SDS PAGE- Determination of molecular weight}

The killer toxin fraction obtained from HPLC was homogeneous on a discontinuous gradient SDS -PAGE with a wide range of molecular mass marker, the toxin moved in a single band near the ovalbumin. The molecular mass of the toxin was $42.6 \mathrm{kDa}$ (Figure 1).

\section{W. anomalus killer toxin assay}

A total of 336 candida isolates were screened for killer toxin assay against Wickerhamomyces anomalous along with standard strain of Saccharomyces cerevisiae. Candida isolates 292 (87\%) were sensitive to the Wickerhamomyces anomalous killer toxin (Figure 2).

\section{Temperature and $\mathrm{pH}$ stability of $W$. anomalus killer toxin}

The killer activity of $W$. anomalus was higher in low temperature and at low $\mathrm{pH}$ but as temperature and $\mathrm{pH}$ increased killer activity decreased. Killer activity was highest at $\mathrm{pH} 5.5$ with temperatures $25{ }^{\circ} \mathrm{C}$ and lowest at $\mathrm{pH} 6$ and $30{ }^{\circ} \mathrm{C}$.

Cell killing kinetics of $W$. anomalus killer toxin against Candida species

Cell killing activity was started with in the first 2 hours for all of the tested Candida strain. Complete cell death was observed after 6 hours, in all species except $C$. tropicalis at 4 hours.

\section{DISCUSSION}

Candidiasis is the most common opportunistic fungal infection. In the past three decades with the use of potent antibacterial, immunesuppressive drugs and advancement in medical practices increased the frequency of invasive candidiasis $[16,17]$. The treatment of fluconazole resistance is extremely difficult with limited options of antifungals [18]. Prolonged therapy and increased use of antifungals for recurrent candidiasis are the most common risk factors for 
azoles resistance in vulvo-vaginitis candidiasis [19-21]. The advancement of antifungal resistance does not give off an impression of being an issue amid their fleeting use, yet is a chief concern for long term treatments [22].

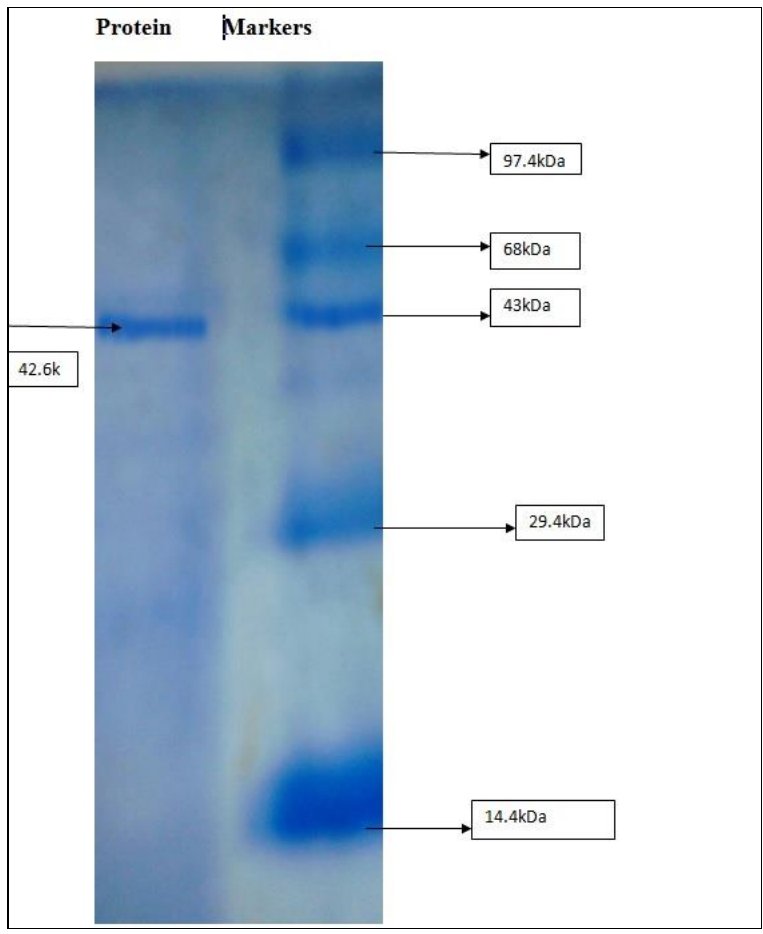

Figure 1. SDS -PAGE analysis of $W$. anomalus Killer toxin protein.

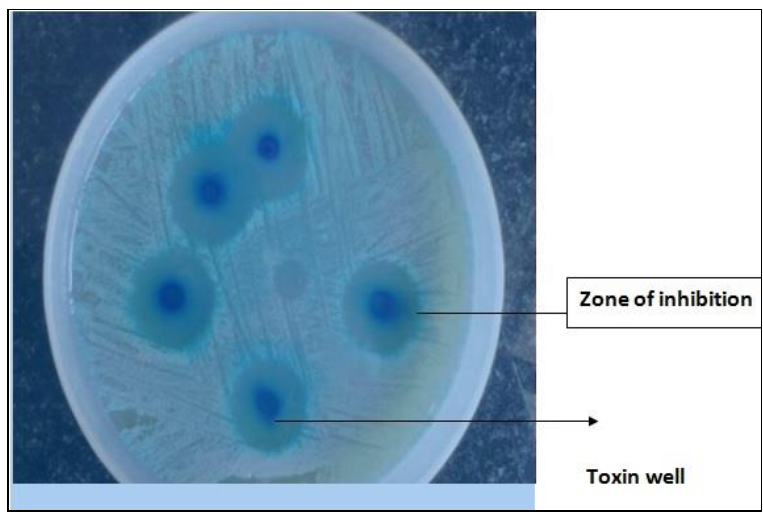

Figure 2. Agar well diffusion assay of Killer toxin.

Killer toxins are reported to have the potential for treatment of fungal infections, which are distributed in several environmental sources. In this study, we aimed to isolate and characterize the yeast killer protein secreted by the yeast $W$. anomalus. Several species of environmental yeasts Wickerhamomyces species have been reported to have the killer character [23]. In our study, we determined the optimum culture conditions for the production of $W$. anomalus killer toxin protein with molecular weight 42.6 $\mathrm{kDa}$ with a single band, estimated by sodium dodecyl sulfate polyacrylamide gel electrophoresis (SDS-PAGE). This appears to be another rare finding of our study that a new killer toxin is identified from $W$. anomalus species which is susceptible to pathogenic Candida species. These findings are in agreement with other studies characterized different types of killer yeasts having molecular mass K1 (20 kDa), K2 (21 kDa), K3 (42 kDa), PaKT (105 kDa) type killer proteins [24,25].The present study demonstrated a different killer toxin from $W$. anomalus species rather than the previous discoveries. The diversity of killer proteins of $W$. anomalus with molecular masses ranging from $8 \mathrm{kDa}$ to more than $300 \mathrm{kDa}$ is already established $[26,27]$, however, this wide range in protein size may arise mainly from different levels of glycosylation.

The protein isolated from $W$. anomalus exhibited basic characteristics of mycocin with $\mathrm{pH} 5.5$ and temperature ranging from 4 to $25^{\circ} \mathrm{C}$. One of the most important aspects of this study is the good activity $(87 \%)$ of killer toxin against the pathogenic strains of Candida species including both clinical and standard isolates tested which correlates with the antagonistic action of $W$. anomalus (WC65, NCYC 432, 434, YF07b) killer toxin against other fungus [28].

This paper validates the observation that different killer toxins are produced by different isolates of the same species. The susceptibility of pathogenic yeasts to killer toxin produced by yeasts, belonging to the genera Wickerhamomyces could lead to the design of synthetic derivatives to be used as antifungal agents and further studies.

The limited number of antifungal drugs with fewer side effects has increased the incidence of candidial infection. So there is a need to search for new products with the effective antifungal abilities without adverse side effects. Environmental yeast killer toxin $W$. anomalus possessed broad antimycotic activity against pathogenic Candida and non-albicans Candida species and can be proposed as a novel 
antimycotic agent for candidiasis. Purified $W$. anomalus killer toxin is identified as a protein having a molecular weight of $42.6 \mathrm{kDa}$. It has a wide range of $\mathrm{pH}(3.5-5.5)$ and temperature (4$\left.25^{\circ} \mathrm{C}\right)$. The $\mathrm{pH} 5.5$ and temperature $(25 \mathrm{oc})$ were appropriate for the use of this toxin in medical and fermentation industry.

Further studies are needed to elaborate an easy-to-use technique for killer toxin which could be used in medicine and which will open its use as an antimicrobial agent and powerful bio control agents in food preservation and fermentation industry. However, the identification of killer characteristics and strain maintenance are labor and cost consuming because the killer characteristics are often controlled by plasmids. Therefore, thorough studies are needed in future regarding the relative stability of purified killer toxins after long term storage, including the establishment of the actual time course of their possible degradation or loss of effectiveness.

\section{ACKNOWLEDGMENTS}

Special thanks to Dr. T. V. Rao, Former Prof and Head of Department, Department of Microbiology, Travancore Medical College, Kollam, Kerala, India

Declaration of Conflicting Interests: The authors declare that they have no conflict of interest.

Funding: This project received no specific grant from any funding agency in the public, commercial, or not-for-profit sectors.

\section{REFERENCES}

1. Nayman AS, Zgune AY, Ertem OT, Erben N, Doyuk KE. Evaluation of risk factors in patients with candiduria. Mikrobiyol Bul 2011; 45: 318-324.

2. Sievert DM, Ricks P, Edwards JR.Antimicrobialresistant pathogens associated with healthcareassociated infections: summary of data reported to the National Healthcare Safety Network at the Centers for Disease Control and Prevention, 2009-2010. Infect Control Hosp Epidemiol 2013; 34:1-14.

3. Pfaller MA, Diekema DJ. Epidemiology of invasive candidiasis: a persistent public health problem. Clin Microbiol Rev 2007; 20(1):133-63.
4. Kothari A, Sagar V. Epidemiology of Candida blood stream infections in a tertiary care institute in India. IJMM 2009; 27:171-172.

5. Bijender KB, Sharma S, Construction of killer industrial yeast Saccharomyces -cerevisiaehau-1 and its fermentation performance. BJM 2010; 41: 477-485.

6. Baeza ME, Sanhueza MA, Cifuentes VH. Occurrence of killer yeast strains in industrial and clinical yeast isolates. Biol Res 2008; 41: 173-182.

7. Wang XZ, Chi L, Yue JL. Purification and Characterization of killer toxin from a marine yeast Pichia anomala YF07b against the pathogenic yeast in crab. Curr Microbiol 2007; 55:396-401.

8. Jagadish C, Nidhi S, Shailpreet KS, et al. Epidemiology of Candida blood stream infections: experience of a tertiary care centre in North India. $J$.Infect Dev Ctries 2013; 7:670-675.

9. Clinical and Laboratory Standards Institute: Reference Method for Broth Dilution Antifungal Susceptibility Testing of Yeasts; Approved Standard- Second Edition M27-A2. National Committee for Clinical Laboratory Standards, Wayne, Pennsylvania, USA 2002.CLSI M27-A3.

10. Branchini ML, Pfaller MA, Rhine et al. Genotype variation and slime production among blood and catheter isolates of $\mathrm{C}$ Parapsilosis. J Clin Microbiol 1994; 32:452-6.

11. Peng $\mathrm{Y}$, Chi ZM, Wang $\mathrm{XH}$, Li J. Purification and molecular characterization of exo- $\beta-1$, 3 glucanases from the marine yeast WilliopsissaturnusWC91-2. Appl Microbiol Biotechnol 2009; 85: 85-94.

12. Izgu $F$, Altinbay $D$, Tureli AE. In vitro activity of Panomycin a novel exo- $\beta-1$, 3-glucanase isolated from Pichia anomala NCYC 434, against Dermatophytes. Mycoses 2006; 50: 31-34.

13. Irvine GB and Shaw C. "High-performance gel permeation chromatography of proteins and peptides on colums of TSK-G2000-SW and TSKG3000SW: A volatile solvent giving separation based on charge and size of polypeptides" Anal Biochem 1986; 155:141-148.

14. Vesterberg O. "Isoelectric focusing of proteins", Electrophoretic Procedures 1971; 22:389-411.

15. Izgu F, Altınbat D, Sertkaya A. Enzymic activity of the K5 type yeast killer toxin and its characterization. Biosci Biotechnol Biochem 2005; 69:2200-2206

16. Menza N, Wanyoike W, Muturi WM. Prevalence of Vaginal Candidiasis and Determination of the Occurrence of Candida Species in Pregnant Women Attending the Antenatal Clinic of Thika District Hospital, Kenya. Open J Med Microbiol 2013; 3: 264-272. 
17. Saldanha DR, Shivanand D. Candida speciation and anti-fungal susceptibility. J Clinic Diagnostic Research 2011; 5: 1177-1181.

18. Mokaddas E, Khan ZU \& Ahmad S. Prevalence of Candida dubliniensis among cancer patients in Kuwait: a 5-year retrospective study. Mycoses 2011; 54, 29-34.

19. Ooga1 VB, Gikunju JK, Bii CC. Characterization and antifungal drug susceptibility of clinical isolates of Candida species. Afr J Health Sci 2011; 19:84-92.

20. Kothari A and Sagar V. Epidemiology of Candida bloodstream infections in a tertiary care institute in India. Indian J Med Microbiol 2009; 27:171-172.

21. Lortholary O, Desnos OM, SitbonK. Recent exposure to caspofungin or fluconazole influences the epidemiology of candidemia: a prospective multicenter study involving 2,441 patients. Antimicrob Agents Chemother 2011; 55:532-8.

22. Finkel JS and Mitchell AP. Genetic control of Candida albicans biofilm development. Nat Rev Microbiol 2011; 9: 109-118.

23. Tavleen J, Urhekar A, Chitra P. Study of Candida species in various clinical samples in a tertiary care hospital. DHR Intern J Med Scien 2014; 5:2.

24. Marcelo EB, Mario AS and Víctor HC. Occurrence of killer yeast strains in industrial and clinical yeast isolates. Biol Res 2008; 41:173-182.

25. Paluszynski JP, Klassen R, Meinhardt F. Pichia acacia killer system: genetic analysis of toxin immunity. Appl Environ Microbiol 2007; 73: 43734378.

26. Pietro B, Benedetta T and Ann EV. The use of killer sensitivity patterns for biotyping yeast strains the state of the art, potentialities and limitations. FEMS Yeast Res 2007; 7:749-760.

27. Gulbiniene G, Kondratiene I, Jokantaite T. Occurrence of killer yeast strains in fruit and berry Wine yeast populations. Food technol biotechnol 2004; 42:159-163.

28. Santos A, Mauro M, Bravo E. PMKT2, a new killer toxin from Pichia membranifaciens, and its promising biotechnological properties for control of the spoilage yeast Brettanomyces bruxellensis. Microbiol 2009; 155: 624-634. 